\title{
Delusional content at initial presentation to a catchment-based early intervention service for psychosis
}

Ann-Catherine Lemonde, Ridha Joober, Ashok Malla, Srividya N. Iyer, Martin Lepage, Patricia Boksa and Jai L. Shah

\section{Background}

During a psychotic episode, patients frequently suffer from severe maladaptive beliefs known as delusions. Despite the abundant literature investigating the simple presence or absence of these beliefs, there exists little detailed knowledge regarding their actual content and severity at the onset of illness.

\section{Aims}

This study reports on delusions during the initiation of indicated treatment for first-episode psychosis (FEP).

\section{Method}

Data were systematically collected from a sample of 636 patients entering a catchment-based early intervention service for FEP. The average severity and frequency of each delusional theme at baseline was reported with the Scale for the Assessment of Positive Symptoms. Delusional severity (globally and per theme) was examined across a number of sociodemographic and clinical variables.

\section{Results}

Delusions were present in the vast majority of individuals experiencing onset of FEP (94\%), with persecutory $(77.7 \%)$ being the most common theme. Persecutory delusions remained consistent in severity across diagnoses, but were more severe with older age at onset of FEP. No meaningful differences in delusional severity were observed across gender, affective versus non-affective psychosis, or presence/absence of substance use disorder. Globally, delusion severity was associated with anxiety, but not depression. Delusions commonly referred to as passivity experiences were related to hallucinatory experiences.

\section{Conclusions}

This community sample offers a rare clinical lens into the severity and content of delusions in FEP. Although delusional severity was consistent across certain sociodemographic and clinical variables, this was not always the case. Future research should now consider the course of delusion themes over time.

\section{Keywords}

Delusions; thought content; first-episode psychosis; symptomatology; early intervention.

\section{Copyright and usage}

(c) The Authors 2020. Published by Cambridge University Press on behalf of the Royal College of Psychiatrists.
In psychiatric settings, delusions have traditionally been defined as false but fixed beliefs even in the presence of clear and contradictory evidence. ${ }^{1}$ There is a long history of examining delusional content, ${ }^{2}$ which is known to reflect socially salient themes (e.g. persecution, grandiosity) and typically cannot be explained by one's cultural background alone. Although delusions are a core feature of schizophrenia, they are also seen in other psychotic and psychiatric disorders. More recent literature has demonstrated that defining features of delusions are also apparent in strongly held beliefs seen in healthy individuals (e.g. political ideologies), ${ }^{3}$ leading some to abandon the long-standing definition of false but fixed beliefs, instead focusing on components such as help-seeking, distress and risk. The current debate surrounding the definition of delusions in part indicates the need for improved understanding of this phenomenon.

Well-established delusional systems seen in later episodes, or chronic psychoses, may contain 'secondary' delusions, ${ }^{4}$ whereas the early phases of delusion formation are, at least in theory, more likely to include 'primary' delusions, emerging from intuitions, percepts, memory and atmosphere. ${ }^{4,5}$ However, despite the considerable attention delusions have received in clinical settings, far less has been reported about their underlying content and severity during the formative stages of illness. Investigating delusions in early clinical samples is critical because relatively young, treatment-naïve presentations are less likely to be confounded by the effects of long-term illness or previous interventions. Improved knowledge on the content and severity of delusions may also have implications for diagnostic classification and personalising therapeutic options. ${ }^{6-8}$

\section{Delusions in early psychosis}

The limited work previously undertaken has found relatively high rates of delusions during first-episode psychosis (FEP), with the most common themes being persecutory, referential and grandiose. ${ }^{7,9,10}$ However, this literature is limited by small sample sizes, selected samples and potential confounds (such as treatment effects or lack of clarity regarding antipsychotic medication exposure). A brief Australian report examining delusions in FEP was catchment-based, but had a modest study sample $(N=143)$ and no mention of prior antipsychotic medication use. ${ }^{9}$ Studies with larger samples $(N=245)$ have had disproportionately high numbers of Black men ${ }^{7}$ or focused on nonaffective psychoses alone. ${ }^{10-12}$ In contrast, an inclusive approach that encompasses both non-affective and affective psychoses (e.g. bipolar affective disorder or major depressive disorder with psychotic features) may provide valuable insight into how symptoms such as delusions emerge and are maintained, and whether diagnostic grouping affects these processes: although certain delusional content has been associated with mood, delusions of persecution appear to be broadly dispersed across diagnostic categories in adult patients. ${ }^{6}$ Regarding sociodemographic factors, FEP reports have shown a link between persecutory delusions and older age at onset of FEP, but not gender. ${ }^{7,13}$ In summary, previous literature on delusional content and severity would be notably strengthened by data from a large, representative and well-characterised early psychosis population.

Although the links between symptoms (e.g. anxiety, hallucinations) and persecutory delusions or delusions as a whole have been researched, ${ }^{14}$ few have rigorously examined the association between other delusion themes (e.g. grandiosity, mind reading) 
and symptoms, ${ }^{15,16}$ and even fewer within FEP populations. ${ }^{7}$ For example, a systematic review demonstrated links between depression, anxiety and overall delusion severity; however, they also called for future research to consider specific subtypes of psychotic symptoms experienced. ${ }^{14}$ Furthermore, certain delusion themes that have commonly been considered as passivity experiences and/or thought alienation may emerge and persist differently than other themes. ${ }^{4}$ One indicator is that these experiences appear to have a distinct association with hallucinations. ${ }^{7,15}$ A more detailed view of the association between clinical factors and delusion severity, both globally and per theme, in a larger and more representative sample may improve psychological models and, ultimately, interventions.

\section{Aims}

The aim of this study was to systematically examine and describe delusional content and severity, and its clinical and sociodemographic characteristics, in a large, minimally medicated and catchment-based clinical population of individuals experiencing an affective or nonaffective FEP. We hypothesized that (a) persecutory delusions would be the most common delusion theme across nonaffective and affective psychoses in this representative sample, and that their severity would be positively associated with age at onset of FEP; (b) severity of anxiety and depression would be positively associated with global severity of delusions and (c) that passivity experiences and/or thought alienation (e.g. thought insertion, thought withdrawal, thought broadcasting and being controlled) would be positively correlated with hallucinations.

\section{Method}

\section{Setting}

The study took place at the Prevention and Early Intervention Program for Psychosis (PEPP-Montreal). PEPP-Montreal is open to youths who are experiencing an FEP within a geographically defined catchment area of roughly 350,000 individuals in an urban setting in south-west Montreal, Canada. ${ }^{17}$ There are no competing public or private early intervention services in the same catchment; all individuals identified as needing treatment for an early-phase psychosis are referred to PEPP-Montreal, thus forming a near-treated incidence sample. ${ }^{18}$ Following intake to the service, PEPP-Montreal provides a comprehensive standardised assessment battery, with longitudinal follow-up for 2 years of treatment. Written informed consent was obtained from all patients to participate in an evaluation and care protocol and as part of various longitudinal FEP outcomes studies, approved by the Institutional Review Board for Human Subjects Research of the Douglas Hospital Research Centre. For those under 18 years of age, consent was obtained from parents or guardians.

\section{Study population}

Inclusion criteria for PEPP-Montreal are age 14-35 years at the time of referral, diagnosis of an affective or non-affective psychotic illness with the Structured Clinical Interview for DSM-IV (SCID-IV), and fluency in either English or French. Patients, both in-patient and out-patient, accepted to PEPP-Montreal may have received antipsychotic medications for no more than 30 days before referral. For the present sample, the average number of days on antipsychotic medication before date of entry was 2.6 days, with the mode being 0 days. Exclusion criteria were IQ $<70$, psychotic illness solely related to substance intoxication or withdrawal, or an organic mental disorder. Only data collected at baseline were used for the current analyses.

\section{Table 1 Sociodemographic and clinical characteristics}

$n(\%)$

Sociodemographic variable

Gender

Female

Male

Missing

$191(30.0 \%)$ $444(69.8 \%)$ $1(0.2 \%)$

Age of entry, years

Mean [s.d.]

Range

$23.8[4.75]$

14-35

Relationship status

In partnership

No partnership

Missing

Education level

Completed high school

Did not complete high school

Missing

Visible minority status

White

Black

Asian

Aboriginal

Other

Missing

$66(10.4 \%)$

$562(88.4 \%)$

$8(1.3 \%)$

$405(63.7 \%)$

$194(30.5 \%)$

37 (5.9\%)

$374(58.8 \%)$

$83(13.1 \%)$

$49(7.7 \%)$

$2(0.3 \%)$

$87(13.7 \%)$

$41(6.4 \%)$

Clinical variables

Diagnosis

Affective psychosis

$172(27.0 \%)$

Non-affective psychosis

$412(64.8 \%)$

Missing

Comorbid substance use disorder

$52(8.2 \%)$

Yes

$286(45.0 \%)$

$259(40.7 \%)$

$91(14.3 \%)$

Missing

Days on continuous antipsychotic medication

before referral

Mean [s.d.]

Mode

Missing

$2.6[5.18]$

$116(18.2 \%)$

\section{Instruments and assessments}

Initial assessment interviews took place within 1 month of first intake to the PEPP-Montreal clinical service. Demographic variables collected included assessments for gender (male/female), age of entry, relationship status (in partnership yes/no), education level (completed high school yes/no) and visible minority status. Visible minority status was self-reported as one of six options $(\text { Table } 1)^{19}$ that, for further analysis, was collapsed to create a binary variable for visible minority status (yes/no). Age at onset of FEP and duration of untreated psychosis (DUP) were obtained through the Circumstances of Onset and Relapse Schedule; ${ }^{20}$ DUP was defined as the number of weeks between the onset of threshold-level psychosis and the initiation of antipsychotic medication. The SCID-IV was used to classify each patient's diagnosis as either non-affective (schizophrenia, schizoaffective, delusional disorder, schizophreniform, brief psychotic disorder or psychosis not otherwise specified) or affective psychosis (bipolar disorder type 2, bipolar disorder type $1 \mathrm{manic} /$ depressed/mixed, bipolar disorder not otherwise specified or major depressive disorder) and to determine if the individual had a comorbid substance use disorder. $^{21}$ Antipsychotic medication use (number of days) before date of entry was acquired during the interview, and corroborated with available prescription information in clinical files. ${ }^{22}$

Delusional content was systematically measured with the Scale for the Assessment of Positive Symptoms (SAPS). ${ }^{23}$ The scale measures severity of the following 12 delusion themes: persecutory, jealousy, sin or guilt, grandiose, religious, somatic, reference, being 
controlled, mind reading, thought broadcasting, thought insertion and thought withdrawal. The severity of each delusion theme is rated on a scale from zero to five, as is a global measure of severity. Severity reflects certain aspects of delusional symptoms, such as frequency, complexity, conviction, preoccupation and interference with functioning.

The SAPS was further used to measure the global rating of positive psychiatric symptoms, and separate ratings were compiled for hallucinations, bizarre behaviours and positive formal thought disorder. The Scale for Assessment of Negative Symptoms (SANS) ${ }^{24}$ was used to determine the global severity of negative psychiatric symptoms, along with separate ratings for affective flattening, alogia, avolition/apathy and anhedonia/asociality. Attention was excluded as it reflects cognition, ${ }^{25}$ and this exclusion has been shown to improve the reliability of the SANS ${ }^{26}$ Furthermore, the 'inappropriate affect' item was removed as it has been shown to poorly correlate with the overall subscale score. ${ }^{27}$ Depression was measured with the total score from the Calgary Depression Scale (CDS) for schizophrenia. ${ }^{28}$ Anxiety was measured with the total score from the Hamilton Rating Scale for Anxiety. ${ }^{29}$

All diagnostic and symptom data were collected by rigorously trained research assistants with at least an undergraduate degree in psychology or a related health science field. The SAPS training occurred full-time over a 4- to 6-week period and included orientation, rating videotapes, role-playing, observing experienced staff leading interviews and conducting interviews under supervision. Yearly interrater reliability sessions are held to calculate intraclass correlations, and serve as continuing education for staff. Intraclass correlations for the SAPS have consistently been high over the 15 years of data collection $(0.73-0.80)$, indicating good to excellent reliability. ${ }^{30}$

\section{Statistical analyses}

Data from 686 consenting patients entering the programme between January 2003 and March 2018 were available for analysis. Those with no SAPS or SANS data collected $(n=11$; see Supplementary Table 1 available at https://doi.org/10.1192/bjp. $2020.157)$, over the age of 35 years $(n=1)$, with a purely substance-induced psychosis $(n=9)$ or an IQ $<70 \quad(n=3)$ were excluded from further analyses. Those on medication for $>30$ days before the referral date were not included $(n=26)$, to reduce the potentially confounding effects of prolonged medication exposure on delusional context and severity; nonetheless, a post hoc analysis revealed that there were no significant differences in global delusion severity between those who had taken medication for $>30$ days and those below this cut-off (Supplementary Table 2). Our final sample therefore includes 636 patients.

All statistical testing was performed with SPSS Statistics version 24 for Windows. ${ }^{31}$ Patient characteristics, both sociodemographic and clinical, were summarised with appropriate descriptive analyses. To further ensure that our final sample was representative of the total population, we identified demographic variables for which $>10 \%$ of information was missing, and examined global delusional severity between subgroups with and without that variable present.

Descriptive statistics were also used to report delusion severity and frequency (globally and per theme) and the co-occurrence of themes. To assess frequencies, we used the same threshold for non-remission (SAPS global scores $\geq 3$ ) recommended by the Working Group on Remission in Schizophrenia. ${ }^{32}$ For the most common delusion themes (those with $\geq 100$ patients with SAPS global scores $\geq 3$ ), Mann-Whitney $U$-tests and Spearman rankorder correlations were used to describe patterns between delusion severity (both globally and per theme), and demographic and clinical variables (Supplementary Table 4). A Poisson regression was used to analyse the relationship between global delusion severity and the number of delusion themes endorsed, and to determine if DUP (log transformed) predicts the number of delusion themes present (exponentiated coefficients presented). We further utilized a Spearman rank-order correlation test to demonstrate the relationship between log-transformed DUP and global delusion severity. Where appropriate, confidence intervals were calculated to better represent uncertainty in the results. All analyses were interpreted with the appropriate Bonferroni-corrected alpha levels.

\section{Results}

\section{Sample characteristics}

Sociodemographic characteristics, as well as diagnosis, comorbid substance use disorder, and antipsychotic medication use (days before date of entry) are summarised in Table 1 . Of the 636 patients, 191 were female and 444 were male. The average age was $23.8 \pm 4.75$ years (range $14-35$ years). A total of $65 \%$ were diagnosed with a non-affective psychosis $(n=412)$ and $27 \%$ were diagnosed with an affective psychosis $(n=172)$. Further, $45 \%(n=286)$ of patients had a comorbid substance use disorder. We found no significant differences in global rating of delusion severity when missing data were $>10 \%$ (Supplementary Table 3).

\section{Delusional content}

Using a SAPS global score $\geq 3$ cut-off, representing moderate-tosevere delusions, 598 patients (94\%) experienced at least one type of delusion. The most commons themes, in order, were persecutory, reference and grandiose; the least common themes were thought broadcasting, thought withdrawal and jealousy (Table 2 and Fig. 1). Delusional themes rarely occurred in isolation, with only $11.9 \%$ of patients presenting with one theme (Table 3 and Supplementary Fig. 1).

\section{Delusion severity}

Globally, delusions tended to present at the more severe end of the spectrum, with an overall score that was "marked" (mean 4.05, s.d. 0.96; Supplementary Fig. 2). Per theme, however, there were noticeable differences in the distribution of severity (see Supplementary Fig. 3). Although persecutory and referential delusions were leftskewed, most other themes presented as right-skewed distributions.

\section{Delusions and sociodemographic variables}

Older age at onset of FEP was associated with more severe global $\left(\boldsymbol{r}_{s}=0.15,95 \%\right.$ CI $\left.0.06-0.22\right)$ and persecutory delusions $\left(\boldsymbol{r}_{s}=0.12\right.$, 95\% CI $0.03-0.20$ ), but not delusions of reference, grandiosity, religiosity, mind reading, being controlled and somatic. Visible minority status and gender were not significant predictors of global or thematic delusion severity (Supplementary Table 4).

\section{Delusions and clinical variables}

Analyses of global or thematic delusional severity with respect to affective versus non-affective psychosis, or presence/absence of substance use disorder, revealed no differences (Supplementary Table 4); similarly, the severity of all delusion themes, including persecutory, remained consistent across non-affective and affective psychoses.

The global rating for delusion severity was positively associated with anxiety, but not with depression. Anxiety was positively associated with persecutory, referential, control and somatic delusions, but not with grandiose, religious or mind reading delusions. 
Table 2 Descriptive statistics of Scale for the Assessment of Positive Symptoms (SAPS) delusion items

\begin{tabular}{|c|c|c|c|c|}
\hline SAPS item delusion & Mean SAPS severity rating $0-5$ & $95 \% \mathrm{Cl}$ for mean & SAPS severity rating $\geq 3, n(\%)$ & $95 \% \mathrm{Cl}$ for percentage \\
\hline Global rating & 4.05 & $3.98-4.12$ & $598(94.0 \%)$ & $91.0-95.7 \%$ \\
\hline Persecutory & 3.44 & 3.33-3.55 & 494 (77.7\%) & $74.2-80.9 \%$ \\
\hline Reference & 2.86 & $2.73-2.99$ & $413(64.9 \%)$ & $61.1-68.7 \%$ \\
\hline Grandiose & 1.83 & $1.69-1.97$ & $256(40.3 \%)$ & $35.4-44.2 \%$ \\
\hline Religious & 1.27 & $1.14-1.40$ & $177(27.8 \%)$ & $24.4-31.5 \%$ \\
\hline Mind reading & 1.09 & $0.98-1.21$ & $150(23.6 \%)$ & $20.3-27.1 \%$ \\
\hline Being controlled & 1.01 & $0.89-1.13$ & $125(19.7 \%)$ & $16.6-23.0 \%$ \\
\hline Somatic & 0.92 & $0.80-1.04$ & $107(16.8 \%)$ & $14.0-20.0 \%$ \\
\hline Guilt or sin & 0.88 & $0.78-0.98$ & 89 (14.0\%) & $11.4-16.9 \%$ \\
\hline Thought insertion & 0.67 & $0.57-0.77$ & $88(13.8 \%)$ & $11.3-16.8 \%$ \\
\hline Thought Broadcasting & 0.56 & $0.47-0.65$ & 72 (11.3\%) & $9.0-14.0 \%$ \\
\hline Thought Withdrawal & 0.33 & $0.26-0.40$ & 38 (6.0\%) & $4.3-8.1 \%$ \\
\hline Jealousy & 0.23 & $0.17-0.29$ & 21 (3.3\%) & $2.1-5.0 \%$ \\
\hline
\end{tabular}

Although there was no association between depression and global delusion severity, depression was positively associated with the severity of referential, mind reading, control and somatic delusions. Grandiose delusions were negatively associated with depression, whereas persecutory and religious delusions had no associations with depression (Table 4).

Mind-reading delusions and delusions of control were positively correlated with hallucinations. Somatic delusions were the only other delusional theme associated with hallucinations.

For every one-unit increase in global delusion severity on the SAPS, the number of delusional themes present increased by 1.47 (95\% CI 1.32-1.56, $P<0.001$ ). However, log-transformed DUP was not associated with the global rating of delusion severity at baseline $\left(\boldsymbol{r}_{\mathrm{s}}=0.04,95 \% \mathrm{CI}-0.05\right.$ to $\left.0.12, P>0.05\right)$, nor with the number of delusion themes present $(B=0.97,95 \%$ CI $0.91-1.03, P>0.05)$.

\section{Discussion}

In this inventory of systematically collected delusional content and severity in a catchment-based and medication-naïve sample of patients with FEP who were entering early intervention services, the vast majority of individuals presented with delusions of moderate severity, and $80.7 \%$ had at least two delusions. As hypothesised, persecutory delusions were the most common theme; these remained consistent in severity across affective and nonaffective diagnoses, and tended to be more severe with older age at onset

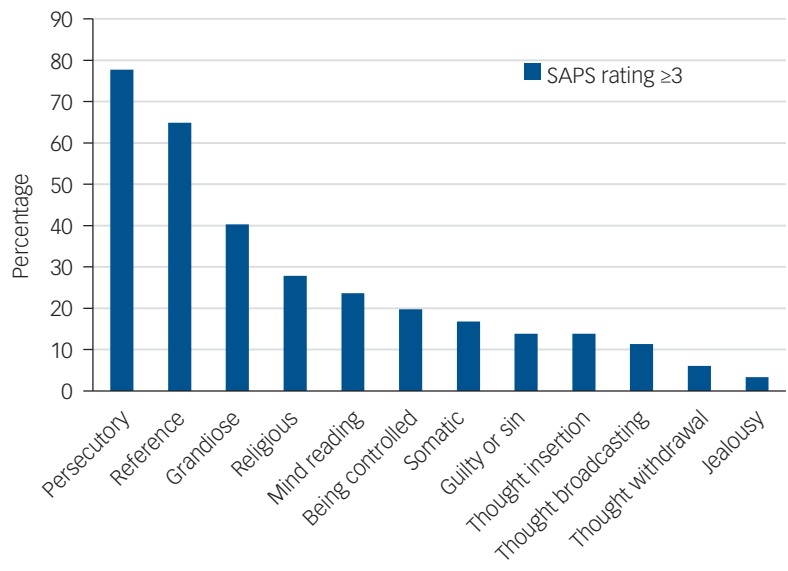

Delusion themes

Fig. 1 Percentage of participants with FEP with a specific delusion theme. SAPS, Scale for the Assessment of Positive Symptoms. of FEP. Global severity of delusions was associated with anxiety but not with depression, with specific relationships emerging per theme. Finally, we found that certain delusional themes that are commonly considered as passivity experiences and/or thought alienation were correlated with hallucinatory experiences.

Our results confirm that persecutory delusions are indeed the most common delusion theme in this near-treated incidence sample of patients with FEP. Unlike the selective samples, potential confounding owing to treatment effects, and/or varying levels of chronicity seen in previous reports, ${ }^{7,9,10,15,16}$ this early-stage, catchment-based clinical population offers a unique context for the examination of delusions. It has been hypothesised that persecutory delusions are a maladaptive defence mechanism in response to chronic environmental stress. ${ }^{7}$ Corlett et al suggested that paranoia and persecutory ideation arise from aberrant prediction errors that create waves of fear and hypervigilance, ${ }^{8}$ whereas Gold and Gold explain paranoia in a social-evolutionary framework that accounts for the high prevalence of socially salient themes. ${ }^{33}$ It may be that persecutory beliefs emerge in an atmosphere of tension, uncertainty and uneasiness, which often accompanies the prodromal period of $\mathrm{FEP}^{3,5}$ and the potential fear and/or stigma associated with this.

As hypothesized, persecutory delusions remained consistent in severity across affective and non-affective diagnoses. Picardi et al similarly found that persecutory and somatic delusions were evenly distributed across diagnoses. ${ }^{6}$ However, their consideration of the polarity of mood within affective psychoses may explain their finding that grandiose delusions varied over diagnostic categories. Similar patterns in delusional themes across diagnoses demonstrates the potential utility of examining their development

\section{Table 3 Descriptive statistics for multiple delusion themes}

$\begin{array}{lrc}\begin{array}{l}\text { Number of } \\ \text { delusion themes }\end{array} & \text { SAPS rating } & 95 \% \mathrm{Cl} \text { for } \\ 0 & \geq 3, n(\%) & \text { percentage } \\ 1 & 37(5.8 \%) & 4.1-7.9 \% \\ 2 & 76(11.9 \%) & 9.5-14.7 \% \\ 3 & 141(22.2 \%) & 19.0-25.6 \% \\ 4 & 137(21.5 \%) & 18.4-24.9 \% \\ 5 & 98(15.4 \%) & 12.7-18.5 \% \\ 6 & 54(8.5 \%) & 6.4-10.9 \% \\ 7 & 48(7.5 \%) & 5.6-9.9 \% \\ 8 & 16(2.5 \%) & 1.4-4.1 \% \\ 9 & 6(0.9 \%) & 0.3-2.0 \% \\ 10 & 7(1.1 \%) & 0.4-2.3 \% \\ 11 & 4(0.6 \%) & 0.2-1.6 \% \\ \text { Missing } & 2(0.3 \%) & 0.0-1.1 \% \\ \text { SAPS, Scale for the Assessment of Positive Symptoms. } & \end{array}$




\begin{tabular}{|c|c|c|c|c|c|c|c|c|c|c|c|c|}
\hline \multirow[t]{2}{*}{ Symptoms } & \multicolumn{3}{|c|}{ Global rating } & \multicolumn{3}{|c|}{ Persecutory } & \multicolumn{3}{|c|}{ Reference } & \multicolumn{3}{|c|}{ Grandiose } \\
\hline & rho & $95 \% \mathrm{Cl}$ & Significance & rho & $95 \% \mathrm{Cl}$ & Significance & rho & $95 \% \mathrm{Cl}$ & Significance & rho & $95 \% \mathrm{Cl}$ & Significance \\
\hline Anxiety & $0.21 *$ & $0.12-0.29$ & 0.000 & $0.25^{*}$ & $0.17-0.33$ & 0.000 & 0.25 & $0.17-0.33$ & 0.000 & -0.03 & $-0.11-0.06$ & 0.564 \\
\hline Depression & 0.06 & $-0.02-0.13$ & 0.173 & 0.09 & $0.01-0.17$ & 0.026 & 0.12 & $0.05-0.20$ & 0.002 & $-0.17^{\star}$ & $-0.25-0.09$ & 0.000 \\
\hline Hallucinations & 0.10 & $0.02-0.17$ & 0.017 & 0.06 & $-0.02-0.14$ & 0.125 & 0.09 & $0.01-0.17$ & 0.020 & 0.00 & $-0.08-0.08$ & 0.985 \\
\hline Bizarre behaviour & $0.11 *$ & $0.04-0.19$ & 0.004 & 0.08 & $0.00-0.15$ & 0.051 & 0.04 & $-0.04-0.11$ & 0.366 & $0.25^{*}$ & $0.18-0.33$ & 0.000 \\
\hline Positive formal thought disorder & 0.09 & $0.01-0.17$ & 0.021 & -0.06 & $-0.14-0.02$ & 0.125 & 0.09 & $0.01-0.16$ & 0.031 & $0.33^{*}$ & $0.26-0.40$ & 0.000 \\
\hline SANS & -0.04 & $-0.12-0.04$ & 0.313 & 0.05 & $-0.03-0.13$ & 0.220 & -0.02 & $-0.09-0.06$ & 0.702 & $-0.19^{\star}$ & $-0.27-0.12$ & 0.000 \\
\hline Affective flattening & -0.07 & $-0.14-0.01$ & 0.096 & 0.02 & $-0.06-0.09$ & 0.696 & 0.00 & $-0.08-0.08$ & 0.968 & $-0.14^{*}$ & $-0.21-0.06$ & 0.000 \\
\hline Alogia & -0.11 & $-0.18-0.03$ & 0.007 & -0.06 & $-0.14-0.01$ & 0.105 & -0.05 & $-0.12-0.03$ & 0.256 & -0.10 & $-0.17-0.02$ & 0.016 \\
\hline Avolition/apathy & $0.12^{*}$ & $0.05-0.20$ & 0.002 & 0.11 & $0.03-0.18$ & 0.007 & -0.04 & $-0.12-0.04$ & 0.286 & -0.05 & $-0.13-0.03$ & 0.214 \\
\hline \multirow[t]{3}{*}{ Anhedonia/asociality } & 0.10 & $0.02-0.18$ & 0.010 & $0.16^{*}$ & $0.08-0.24$ & 0.000 & 0.04 & $-0.04-0.12$ & 0.344 & $-0.12^{*}$ & $-0.20-0.05$ & 0.002 \\
\hline & \multicolumn{3}{|c|}{ Religious } & \multicolumn{3}{|c|}{ Mind reading } & \multicolumn{3}{|c|}{ Being controlled } & \multicolumn{3}{|c|}{ Somatic } \\
\hline & rho & $95 \% \mathrm{Cl}$ & Significance & rho & $95 \% \mathrm{Cl}$ & Significance & rho & $95 \% \mathrm{Cl}$ & Significance & rho & $95 \% \mathrm{Cl}$ & Significance \\
\hline Anxiety & 0.00 & $-0.09-0.08$ & 0.954 & 0.10 & $0.01-0.18$ & 0.030 & $0.21 *$ & $0.12-0.29$ & 0.000 & $0.22^{*}$ & $0.13-0.30$ & 0.000 \\
\hline Depression & -0.05 & $-0.13-0.03$ & 0.186 & $0.15^{*}$ & $0.07-0.23$ & 0.000 & $0.13^{*}$ & $0.05-0.21$ & 0.001 & $0.12^{*}$ & $0.04-0.20$ & 0.003 \\
\hline Hallucinations & 0.09 & $0.01-0.17$ & 0.022 & $0.24^{*}$ & $0.17-0.32$ & 0.000 & $0.25^{*}$ & $0.17-0.32$ & 0.000 & $0.17^{*}$ & $0.09-0.24$ & 0.000 \\
\hline Bizarre behaviour & $0.20^{*}$ & $0.13-0.28$ & 0.000 & 0.10 & $0.02-0.17$ & 0.015 & $0.17 *$ & $0.09-0.25$ & 0.000 & $0.12^{*}$ & $0.04-0.20$ & 0.002 \\
\hline Positive formal thought disorder & $0.22^{\star}$ & $0.15-0.30$ & 0.000 & 0.04 & $-0.04-0.12$ & 0.319 & 0.03 & $-0.05-0.11$ & 0.435 & 0.09 & $0.01-0.16$ & 0.033 \\
\hline SANS & -0.03 & $-0.11-0.05$ & 0.435 & 0.11 & $0.03-0.18$ & 0.009 & 0.09 & $0.01-0.17$ & 0.028 & $0.15^{*}$ & $0.07-0.23$ & 0.000 \\
\hline Affective flattening & -0.04 & $-0.12-0.04$ & 0.287 & 0.03 & $-0.05-0.10$ & 0.497 & 0.03 & $-0.05-0.10$ & 0.516 & 0.11 & $0.03-0.19$ & 0.006 \\
\hline Alogia & 0.01 & $-0.07-0.09$ & 0.837 & 0.04 & $-0.04-0.12$ & 0.343 & 0.07 & $0.00-0.15$ & 0.065 & 0.10 & $0.02-0.17$ & 0.015 \\
\hline Avolition/apathy & 0.03 & $-0.05-0.10$ & 0.520 & 0.04 & $-0.04-0.11$ & 0.371 & 0.02 & $-0.05-0.10$ & 0.541 & 0.08 & $0.00-0.16$ & 0.042 \\
\hline Anhedonia/asociality & -0.06 & $-0.14-0.02$ & 0.143 & 0.10 & $0.02-0.18$ & 0.011 & 0.03 & $-0.05-0.11$ & 0.466 & $0.13^{*}$ & $0.05-0.21$ & 0.001 \\
\hline
\end{tabular}


and maintenance as transdiagnostic phenomena with common underlying cognitive mechanisms. ${ }^{6}$ Combining this with phenomenological and qualitative analyses that incorporate a more indepth assessment of delusional content may also be important.

Previous studies have reported that persecutory delusions are more common and severe in older patients, ${ }^{13}$ even when investigating early psychosis samples with younger age ranges; ${ }^{7}$ our analyses raise the possibility that this association may be unique to persecutory delusions. Current theories suggest that such delusions are the product of a hyper-inferential state, involving the ability to surmise the intentions of others, a process that is not completely developed by early adolescence. ${ }^{7,34}$ At a neurobiological level, paranoia is postulated to be associated with prediction error dysfunction in certain brain regions, which then plays a role in the ability to infer the intentions of others. ${ }^{8}$ Consistent with our findings, Hafner et al showed that adolescents tend to present with undifferentiated delusions, as opposed to systematised persecutory delusions in later life. ${ }^{13}$

Neurobiological and cognitive models have also suggested that delusional beliefs are continually reconsolidated and strengthened because of aberrations of synaptic plasticity. ${ }^{8,35}$ It is plausible that these extended prediction errors in turn influence the development of more systematised delusions. Thus, a prognostic factor such as DUP might affect the initial development and severity of delusional themes. However, in our study, DUP was not related to the global severity of baseline delusions nor to the number of delusional themes present in FEP. Future work may wish to consider a different proxy for the complexity and systematisation of the delusional system, including incorporating an analysis of the longitudinal course of delusions in early psychosis.

A systematic review previously highlighted that anxiety and depression were related to the severity, distress and content of overall psychotic symptoms. ${ }^{14}$ However, the majority of research has focused on global ratings for delusions and/or solely on persecutory delusions. Although our representative sample and findings further strengthen the notion that anxiety and persecutory delusions are related, this association was not present for grandiose, religious or mind reading themes. Within the context of depression, there are mixed findings regarding the association with persecutory delusions, ${ }^{7,14}$ which may reflect different approaches to assessing symptoms (both persecutory delusions and negative affect). For example, previous studies have included measures of anxiety in composite scores for depression, ${ }^{36,37}$ or examined specific content within the overarching persecutory theme. ${ }^{38,39}$ In addition, although the CDS distinguishes depressive and negative symptoms, previous studies measured depression through selfreport questionnaires. ${ }^{37-39}$ Our sample also included affective psychoses whereas the CDS was designed for schizophrenia and non-affective psychoses, perhaps influencing our findings. While we did not find an association with persecutory delusions, depression was related to other themes (Table 4). Future work may wish to investigate the onset and course of these clinical states over time, to better understand their role in the early development of delusions and to study whether interventions targeting negative affect can mediate delusional severity. ${ }^{40}$

As expected, the severity of mind reading delusions and delusions of control were positively correlated with hallucinations. Passivity experiences, characterised by the belief that one's thoughts or actions are influenced or controlled by an external agent, have traditionally been viewed as delusions. However, unlike other delusional themes, these experiences may be more consistent with perceptual disturbances. ${ }^{41}$ Others have also pointed to a potential source-monitoring bias in which hallucinations and delusions of influence share certain cognitive mechanisms. ${ }^{7}$

\section{Strength, limitations and future directions}

The major strengths of this study are the unique data collected from a large, catchment-based sample with no competing public or private services, meaning that it is a reasonably representative, near-treated incidence sample of all individuals aged 14-35 years who were identified as having FEP in a defined geographic catchment. Furthermore, because of our cut-off of 30 days for medication use, the results are relatively uninfluenced by confounding factors, such as long-term treatment effects or chronicity of illness.

Limitations include the fact that although data were collected as systematically as possible, $20 \%$ of patients receiving care did not provide consent to their data being used for research purposes, and were therefore excluded from this analysis $(n=181)$. Unlike those with missing data, this lack of consent means that we could not examine differences between this group and those included in the study. Symptom assessments also reflect the most acute state of symptoms within the past 3 months, with corresponding potential for recall bias. A total of $21 \%$ of baseline assessments were reconstructed at a later date, using detailed chart notes. Finally, this study is based on data collected prospectively over a 15-year period, opening up the possibly of inconsistencies in data collection as a result of multiple raters. However, PEPP-Montreal minimises these limitations by using standardised assessments, rigorously training research staff and periodically establishing interrater reliability.

Future work may wish to investigate the course of delusions over time, including focusing on specific themes and/or their overlaps. Few have examined how early life factors, such as socioenvironmental context, relate to the content and severity of delusions at initial presentation for FEP. This also involves integrating smaller samples with in-depth, phenomenologically oriented interviews. Investigating associations between delusional content and sociocultural variables in FEP might prove important for identifying groups that are particularly at risk, and could therefore benefit from improved prevention and early intervention efforts.

Ann-Catherine Lemonde (D), BSC, Department of Psychiatry, McGill University Montreal, Quebec, Canada; Ridha Joober. MD, PhD, Department of Psychiatry, McGil University; and Program for Early Intervention and Prevention of Psychoses (PEPPMontreal), Douglas Mental Health University Institute, Quebec, Canada; Ashok Malla (C) MBBS, FRCPC, Department of Psychiatry and Department of Epidemiology and Biostatistics, McGill University; and Program for Early Intervention and Prevention of Psychoses (PEPP-Montreal), Douglas Mental Health University Institute, Quebec, Canada; Srividya N. Iyer, PhD, Department of Psychiatry, McGill University; and Program for Early Intervention and Prevention of Psychoses (PEPP-Montreal), Douglas Mental Health University Institute, Quebec, Canada; Martin Lepage, PhD, Department of Psychiatry, McGill University; and Program for Early Intervention and Prevention of Psychoses (PEPPMontreal), Douglas Mental Health University Institute, Quebec, Canada; Patricia Boksa, PhD, Department of Psychiatry, McGill University; and Douglas Mental Health University Institute, Quebec, Canada; Jai L. Shah, MD, FRCPC, Department of Psychiatry, McGill University; and Program for Early Intervention and Prevention of Psychoses (PEPPMontreal), Douglas Mental Health University Institute, Quebec, Canada

Correspondence: Jai L. Shah. Email: jai.shah@mcgill.ca

First received 7 Jan 2020, final revision 30 Jun 2020, accepted 31 Jul 2020

\section{Supplementary material}

Supplementary material for this paper is available online at http://doi.org/10.1192/bjp.2020.157

\section{Data availability}

Data were requested from the curated database at the Prevention and Early Intervention Program for Psychosis (PEPP-Montreal) at the Douglas Mental Health University Institute. Once access was granted to relevant data, they were and continue to be accessible to al authors in a shared and password-protected drive. This drive is only accessible through servers at the Douglas Mental Health University Institute. 


\section{Acknowledgements}

The completion of this project would not have been possible without technical support from Nicole Pawliuk and Kevin MacDonald regarding data. We sincerely appreciate the support of thesis committee members Dr lan Gold and Dr Geneviève Gariépy.

\section{Author contribution}

A.-C.L. J.L.S., P.B. and A.M. substantially contributed to the conception or design of the manuscript. A.M. S.N. M.L. R. and J.LS. were involved with the curation of the PEPP-Montreal database (data acquisition). A.-C.L. analysed the data under the supervision of J.L.S. and P.B., database (data acquisition). A.-C.L. analysed the data under the supervision of J.L.S. and P.B.,
and all three authors were involved with the interpretation of the data. All authors critically drafted and revised the work for important intellectual content; furthermore, all authors take full accountably for all aspects of the manuscript, and approved the submitted version for publication.

\section{Funding}

This work was supported by the Joseph Armand Bombardier Canadian Graduate ScholarshipMaster's from the Social Sciences and Humanities Research Council of Canada (A.-C.L.), and a student award from the Fonds de Recherche du Quebec-Sante (A.-C.L); and by salary awards from the Canadian Institutes of Health Research (S.N.I.), the Fonds de Recherche du QuebecSanté (R.J., M.L., J.L.S.) and the Canada Research Chairs programme (A.M.)

\section{Declaration of interest}

None

ICMJE forms are in the supplementary material, available online at https://doi.org/10.1192/ bjp.2020.157

\section{References}

1 American Psychiatric Association. Diagnostic and Statistical Manual of Mental Disorders (5th edn). American Psychiatric Association, 2013.

2 Sass LA, Pieknos E. Delusion: the phenomenological approach. In The Oxford Handbook of Philosophy and Psychiatry (eds KWM Fulford, M Davies, RGT Gipps, G Graham, JZ Sadler, G Stanghellini, T Thornton): 632-57. Oxford University Press, 2013.

3 Bortolotti L (ed.). Delusions in context. Palgrave Macmillan, 2018.

4 Oyebode F. Sims' Symptoms in the Mind : Textbook of Descriptive Psychopathology (5th edn). Saunders, 2015.

5 Jaspers K. General Psychopathology: Volume 2. Johns Hopkins University Press, 1997.

6 Picardi A, Fonzi L, Pallagrosi M, Gigantesco A, Biondi M. Delusional themes across affective and non-affective psychoses. Front Psychiatry 2018; 9: 132.

7 Paolini E, Moretti P, Compton MT. Delusions in first-episode psychosis: principal component analysis of twelve types of delusions and demographic and clinical correlates of resulting domains. Psychiatry Res 2016; 243: 5-13.

8 Corlett PR, Taylor JR, Wang XJ, Fletcher PC, Krystal JH. Toward a neurobiology of delusions. Prog Neurobiol 2010; 92: 345-69.

9 Rajapakse T, Garcia-Rosales A, Weerawardene S, Cotton S, Fraser R. Themes of delusions and hallucinations in first-episode psychosis. Early Interv Psychiatry 2011; 5: 254-8.

10 Ellersgaard D, Mors O, Thorup A, Jorgensen $\mathrm{P}$, Jeppesen $\mathrm{P}$, Nordentoft M. Prospective study of the course of delusional themes in first-episode nonaffective psychosis. Early Interv Psychiatry 2014; 8: 340-7

11 Compton MT, Potts AA, Wan CR, Ionescu DF. Which came first, delusions or hallucinations? An exploration of clinical differences among patients with firstepisode psychosis based on patterns of emergence of positive symptoms. Psychiatry Res 2012; 200: 702-7.

12 Vazquez-Barquero JL, Lastra I, Cuesta Nunez MJ, Herrera Castanedo S, Dunn G. Patterns of positive and negative symptoms in first episode schizophrenia. $\mathrm{Br} J$ Psychiatry 1996; 168: 693-701.

13 Hafner $\mathrm{H}$, Maurer $\mathrm{K}$, Loffler W, Riecher-Rossler A. The influence of age and sex on the onset and early course of schizophrenia. Br J Psychiatry 1993; 162: 80-6.

14 Hartley S, Barrowclough C, Haddock G. Anxiety and depression in psychosis: a systematic review of associations with positive psychotic symptoms. Acta Psychiatr Scand 2013; 128: 327-46.

15 Kimhy D, Goetz R, Yale S, Corcoran C, Malaspina D. Delusions in individuals with schizophrenia: factor structure, clinical correlates, and putative neurobiology. Psychopathology 2005; 38: 338-44.
16 Gutierrez-Lobos K, Schmid-Siegel B, Bankier B, Walter H. Delusions in firstadmitted patients: gender, themes and diagnoses. Psychopathology 2001; 34: 1-7.

17 Iyer S, Jordan G, MacDonald K, Joober R, Malla A. Early intervention for psychosis: a Canadian perspective. J Nerv Ment Dis 2015; 203: 356-64.

18 Edwards J, Rodrigues R, Anderson KK. Framing the incidence of psychotic disorders: the case for context. Psychol Med 2019; 49: 2637-8.

19 Maraj A, Veru F, Morrison L, Joober R, Malla A, Iyer S, et al. Disengagement in immigrant groups receiving services for a first episode of psychosis. Schizophr Res 2018; 193: 399-405.

20 Norman RM, Malla AK, Verdi MB, Hassall LD, Fazekas C. Understanding delay in treatment for first-episode psychosis. Psychol Med 2004; 34: 255-66.

21 First MB, Spitzer RL, Gibbon M, Williams JBW. Structured Clinical Interview for DSM-IV-TR Axis I Disorders, Research Version, Patient Edition with Psychotic Screen (SCID-I/P W/PSY SCREEN). New York State Psychiatric Institute, 2002.

22 Cassidy CM, Rabinovitch M, Schmitz N, Joober R, Malla A. A comparison study of multiple measures of adherence to antipsychotic medication in first-episode psychosis. J Clin Psychopharmacol 2010; 30: 64-7.

23 Andreasen NC. Scale for the assessment of positive symptoms. lowa City, University of lowa, 1984.

24 Andreasen NC. Scale for the assessment of negative symptoms. lowa City, University of lowa, 1984.

25 Vadhan NP, Serper MR, Harvey PD, Chou JC, Cancro R. Convergent validity and neuropsychological correlates of the Schedule for the Assessment of Negative Symptoms (SANS) attention subscale. J Nerv Ment Dis 2001; 189: 637-41.

26 Peralta V, de Leon J, Cuesta MJ. Are there more than two syndromes in schizophrenia? A critique of the positive-negative dichotomy. Br J Psychiatry 1992; 161: 335-43.

27 Andreasen NC. Negative symptoms in schizophrenia. Definition and reliability. Arch Gen Psychiatry 1982; 39: 784-8.

28 Addington D, Addington J, Maticka-Tyndale E. Assessing depression in schizophrenia: the Calgary Depression Scale. Br J Psychiatry Suppl 1993; 22 39-44.

29 Riskind JH, Beck AT, Brown G, Steer RA. Taking the measure of anxiety and depression. Validity of the reconstructed Hamilton scales. J Nerv Ment Dis 1987; 175: 474-9.

30 Cicchetti DV. Guidelines, criteria, and rules of thumb for evaluating normed and standardized assessment instruments in psychology. Psychological Assessment 1994; 6: 284-90.

31 SPSS Statistics for Windows, Version 24.0. Armonk, NY: IBM Corp; Released 2016.

32 Andreasen NC, Carpenter WT Jr., Kane JM, Lasser RA, Marder SR, Weinberger DR. Remission in schizophrenia: proposed criteria and rationale for consensus. Am J Psychiatry 2005; 162: 441-9.

33 Gold J, Gold I. Suspicious Minds: How Culture Shapes Madness. Free Press, 2014.

34 Galdos P, van Os J. Gender, psychopathology, and development: from puberty to early adulthood. Schizophr Res 1995; 14: 105-12.

35 Corlett PR, Krystal JH, Taylor JR, Fletcher PC. Why do delusions persist? Front Hum Neurosci 2009; 3: 12

36 Drake RJ, Pickles A, Bentall RP, Kinderman P, Haddock G, Tarrier N, et al. The evolution of insight, paranoia and depression during early schizophrenia. Psychol Med 2004; 34: 285-92.

37 Bentall RP, Rowse G, Shryane N, Kinderman P, Howard R, Blackwood N, et al. The cognitive and affective structure of paranoid delusions: a transdiagnostic investigation of patients with schizophrenia spectrum disorders and depression. Arch Gen Psychiatry 2009; 66: 236-47.

38 Green C, Garety PA, Freeman D, Fowler D, Bebbington P, Dunn G, et al. Content and affect in persecutory delusions. Br J Clin Psychol 2006; 45: 561-77.

39 Chadwick PD, Trower P, Juusti-Butler TM, Maguire N. Phenomenological evidence for two types of paranoia. Psychopathology 2005; 38: 327-33.

40 Opoka SM, Ludwig L, Lincoln TM. A systematic review of trails targeting depression and anxiety in patients with delusions. Zeitschrift für Psychologie 2018; 226: 142-51.

41 Casey P, Kelly B. Fish's Clinical Psychopathology: Signs and Symptoms in Psychiatry. Cambridge University Press, 2019. 